Full metadata for this item is available in Research@StAndrews:FullText at: http://research-repository.st-andrews.ac.uk/

\title{
Cross-species variation in gaze following and conspecific preference among great apes, human infants and adults
}

\author{
Kano, F. \& Call, J.
}

\begin{tabular}{|c|c|}
\hline Date of deposit & 09/03/2016 \\
\hline Version & This is an author version of this work. \\
\hline Access rights & $\begin{array}{l}\text { Copyright (c) } 2014 \text { The Association for the Study of Animal } \\
\text { Behaviour. Published by Elsevier Ltd. All rights reserved. This } \\
\text { work is made available online in accordance with the publisher's } \\
\text { policies. This is the author created, accepted version manuscript } \\
\text { following peer review and may differ slightly from the final } \\
\text { published version. }\end{array}$ \\
\hline $\begin{array}{l}\text { Citation for } \\
\text { published version }\end{array}$ & $\begin{array}{l}\text { Kano, F., \& Call, J. (2014). Cross-species variation in gaze } \\
\text { following and conspecific preference among great apes, human } \\
\text { infants and adults. Animal Behaviour, } 91,137-150 .\end{array}$ \\
\hline $\begin{array}{l}\text { Link to published } \\
\text { version }\end{array}$ & http://dx.doi.org/10.1016/j.anbehav.2014.03.011 \\
\hline
\end{tabular}


1 Although previous studies have shown that many species follow gaze, few study directly

2 compared between closely related species, and thus its cross-species variation remains

3 largely unclear. In this study, we compared between three great ape species (bonobos,

4 Pan paniscus, chimpanzees, Pan troglodytes, orangutans, Pongo abelii) and humans

5 (12-month-olds and adults) in their gaze-following responses to the videos of

6 conspecific and allospecific models. In the video, the model turned his head repeatedly

7 to one of the two identical objects. We used a non-invasive eye-tracking technique to

8 measure participants' eye movements, and used both conspecific and allospecific

9 models as stimuli to examine their potential preference in following conspecific rather

10 than allospecific gaze. Experiment 1 presented to great apes the videos of conspecific

11 and human models. We found that all species follow the conspecific gaze.

12 Chimpanzees did not follow the human gaze, while bonobos did. Bonobos reacted

13 overall more sensitively than chimpanzees to both conspecific and human gaze.

14 Experiment 2 presented to human infants and adults the videos of human, chimpanzee

15 and orangutan models. Both infants and adults followed the human gaze. Unlike adults,

16 infants did not follow the ape gaze. Experiment 3 presented to great apes the videos of

17 allospecific ape models. Consistent with Experiment 1, chimpanzees did not follow the

18 allospecific ape gaze, while bonobos and orangutans did. Importantly, preferential

19 following of conspecific gaze by chimpanzees (Experiment 1) and human infants

20 (Experiment 2) was mainly explained by their prolonged viewing of conspecific face.

21 Thus, it seems to reflect their motivation to selectively attend to the conspecific models.

22 Taken together, we conclude that, gaze following is modulated by both Subject species

23 and Model species in great apes and humans, presumably a reflection of the subjects'

24 intrinsic sensitivity to gaze and also their selective interest in particular models. 
Keywords; conspecific model, gaze following, great apes, human infants, species difference

Gaze following, defined as looking in the same direction as others after seeing their gaze direction, is one of the best studied social behaviours in comparative cognition. Gaze following functions in various ways depending on the species and contexts; from simply exploiting the same information that others have acquired to making inferences about others' intentions and knowledge (Hare, Call, \& Tomasello, 2000). Gaze following has been documented in numerous species, including primates [great ape: (Bräuer, Call, \& Tomasello, 2005); Old World monkeys: (Anderson \& Mitchell, 1999; Emery, Lorincz, Perrett, Oram, \& Baker, 1997; Scerif, Gomez, \& Byrne, 2004); New World monkeys: (Amici, Aureli, Visalberghi, \& Call, 2009; Burkart \& Heschl, 2006), lemurs (Ruiz, Gómez, Roeder, \& Byrne, 2009; Sandel, MacLean, \& Hare, 2011; Shepherd \& Platt, 2008)], nonprimate mammals [dogs: (Téglás, Gergely, Kupán, Miklósi, \& Topál, 2012); goats (Kaminski, Riedel, Call, \& Tomasello, 2005)], birds [ravens: (Bugnyar, Stöwe, \& Heinrich, 2004); bald ibises: (Loretto, Schloegl, \& Bugnyar, 2010)], and reptiles (Wilkinson, Mandl, Bugnyar, \& Huber, 2010). Although gaze following appears quite widespread in phylogeny, studies have also documented its variation among closely related species. Thus, stumptailed macaques follow gaze more frequently than other macaque species (Tomasello, Call, \& Hare, 1998), bonobos more than chimpanzees (Herrmann, Hare, Call, \& Tomasello, 2010) and human children more than great apes (Herrmann, Call, Hernandez-Lloreda, Hare, \& Tomasello, 2007), especially when only the model's eyes (not the head direction) served as a gaze cue (Tomasello et al., 2007). 
Moreover, rather than simply co-orienting with the model, in more complex

settings where individuals have to take into account the position and nature of visual barriers in relation to both the model and themselves, the distribution of gaze following among species appears more restricted. Thus, following gaze around barriers has been documented in apes, ravens, capuchin and spider monkeys but not in marmosets and bald ibises (Amici, et al., 2009; Bräuer, et al., 2005; Bugnyar, et al., 2004; Loretto, et al., 2010; Tomasello, Hare, \& Agnetta, 1999). Moreover, bonobos and chimpanzees, unlike orangutans, take barrier opacity into consideration when following the gaze of others (Okamoto-Barth, Call, \& Tomasello, 2007) and double-looks (i.e., looking back at the model's face after following her gaze and detecting nothing remarkable) have been observed in great apes and Old world monkeys but not in capuchin and spider monkeys (Amici, et al., 2009; Bräuer, et al., 2005; Scerif, et al., 2004).

Taken together, these studies show that even though the presence of gaze following is displayed by numerous species, its expression in terms of strength and flexibility vary substantially among species. Data like these are crucial to be able to test evolutionary hypotheses linking gaze following with social and ecological factors that may contribute to explain the differences among species, including the differences between human and nonhuman animals (Rosati \& Hare, 2009). However, this sort of evolutionary analysis is currently hindered by two major difficulties. First, gaze following is modulated not only by the individuals' potential abilities but also by motivational or contextual factors. For example, in the previous studies with macaque species, the subjects preferentially followed the gaze of particular individuals depending on the social relationship with, and emotional status of the model (Goossens, Dekleva, Reader, Sterck, \& Bolhuis, 2008; Micheletta \& Waller, 2012; Shepherd, Deaner, \& Platt, 
2006; Teufel, Gutmann, Pirow, \& Fischer, 2010). Most relevant for the species comparison is that many previous studies have used human models rather than conspecific models for pragmatic reasons, and thus it is possible that the species differ in the sensitivities to only human but not conspecific gaze. For example, Hattori, Kano, \& Tomonaga (2010) found that chimpanzees followed the gaze of a conspecific but not of a human model when they were presented with the still pictures of those models (but see Itakura, Agnetta, Hare, \& Tomasello (1999), while human adults followed the gaze of both types of models. Ideally, when comparing between two or more species, one should use a crossed design with two factors: Subject species and Model species; i.e. presenting the models of both species to the subjects of both species.

Second, the dependent measure most often used in previous studies has been head turning frequency due to the difficulty in recording the eye movements directly. However, species may differ in their physical constraints to move their head, body, and eyes. For example, orangutans frequently move eyes but not heads to shift their gaze (i.e. sideway gaze) (Kaplan \& Rogers, 2002). Therefore, additional measurements based on eye direction alone may reveal gaze following that goes undetected when using more coarse measures based on head turning.

The developmental differences should also be taken into consideration when comparing between species, especially between species that may follow different developmental trajectories. Previous studies have shown that the sensitivity and flexibility of gaze following change with age in human and nonhuman primates. That is, human infants begin to follow the gaze of others from 3-6 month of age (D'Entremont, Hains, \& Muir, 1997; Hood, Willen, \& Driver, 1998) and establish a robust pattern from 1 year of age (Corkum \& Moore, 1998; von Hofsten, Dahlstrom, \& Fredriksson, 2005). 
Moreover, around 1 year of age human infants begin to follow gaze geometrically to regions beyond their immediate view (Moll \& Tomasello, 2004). Similarly to nonhuman primates, human infants' gaze following is modulated by the motivational and contextual factors. For example, they preferentially follow the gaze of those who have looked

101 toward interesting things versus nothing in the past (Chow, Poulin - Dubois, \& Lewis, 102 2008), and take into account whether individuals have their eyes opened or closed

103 (Brooks \& Meltzoff, 2002). In non-human primates, studies have shown that 104 macaques and chimpanzees begin to follow gaze by around one and three years of age, 105 respectively, and continue to increase the frequency of gaze following with age (Ferrari, 106 Kohler, Fogassi, \& Gallese, 2000; Tomasello, Hare, \& Fogleman, 2001) (but see

107 Okamoto et al. 2002 for the earlier onset of gaze following in a chimpanzee). Moreover, macaques and chimpanzees display a relatively late onset for voluntary control of gaze following such as habituation to unreliable observers (Tomasello et al. 2001) and 110 double-looks (Braeuer et al., 2005). This study aimed to reveal the variation of gaze following among 112 closely-related species by addressing the above-mentioned issues. We used a 113 crossed design with two factors, Subject species and Model species, and studied four 114 hominid species, bonobos (Pan paniscus) chimpanzees (Pan troglodytes) orangutans 115 (Pongo abelii) and human infants (12-month-olds) and control adults (Homo sapiens) 116 (Figure 1). We implemented a relatively simple setting to examine the basic 117 performances of gaze following among species. That is, we measured the frequency 118 of gaze following when each species was observing a human or conspecific model 119 repeatedly turning his head to one of the two identical objects. We adopted the 120 eye-tracking method for two reasons: (1) to present controlled gaze cues of both 
conspecific and allospecific models on the computer monitor and (2) to rely on the eye

122 movement measurement which is relatively independent of physical constraints. We examined whether species (1) showed any evidence of gaze following for each model species, (2) differentiated between conspecific and allospecific gaze, and (3) differed from one another in their overall gaze sensitivities (frequency and/or response time) when presented with either conspecifics or allospecifics. A previous study confirmed that the great apes did not differ from one another in their basic patterns of eye movement (Kano, Hirata, Call, \& Tomonaga, 2011). However, the same previous study also confirmed that humans, especially infants (Hood \& Atkinson, 1993) tend to shift their gaze less frequently (the fixations were "stickier") than apes. Due to this species difference and some procedural differences that existed for pragmatic reasons (e.g. the type of attracting stimuli), we did not compare between great apes and humans in a single experiment. Experiment 1 presented to great apes the videos of conspecific and human model. Experiment 2 presented to human infants and adults the videos of human and allospecific ape model (chimpanzee and orangutan). Experiment 3 returned to great apes and presented the videos of allospecific ape models. 
143 Experiment 1

144 We examined the gaze following responses in bonobos, chimpanzees, and orangutans

145 when they were presented with a conspecific or a human model repeatedly turning his

146 head to one of the two identical objects. Based on previous studies using eye-tracking

147 (Hattori, et al., 2010), we predicted that chimpanzees would preferentially follow the

148 conspecific gaze rather than the human gaze. In addition, based on previous studies

149 using a different behavioural paradigm (Bräuer, et al., 2005; Herrmann, et al., 2010), we

150 predicted that bonobos would follow gaze, at least the human gaze, more frequently

151 than chimpanzees. Finally, based on previous studies using behavioural paradigms

152 (Bräuer, et al., 2005; Okamoto-Barth, et al., 2007), we predicted that orangutans would

153 follow the gaze of either conspecific or human models; however, it was unclear whether

154 they would follow gaze differentially depending on the observed species.

155

156

Method

157 Participants

158 Eight bonobos (Pan paniscus), 14 chimpanzees (Pan troglodytes), and 7 orangutans

159 (Pongo abelii) participated (mean age 16.5 years; 16 females, 13 males). All apes lived in groups (> 10 individuals) with their conspecifics (but not with their allospecifics) in the Wolfgang Köhler Primate Research Centre (WKPRC). Most of the apes were raised by their biological mothers. Although some of them were reared by humans early in ontogeny (hand reared), they mostly grew up with conspecifics from an early age (for the details about participants, see Table A1). All great apes were housed in semi-natural indoor enclosures (175-430 $\left.\mathrm{m}^{2}\right)$ with sleeping and testing rooms, and also in outdoor enclosures during the summer time $\left(1400-4000 \mathrm{~m}^{2}\right)$. Both enclosures were equipped 
with climbing structures, natural vegetation, and enrichment devices to foster extractive foraging activities. They were provided with fresh fruits, vegetables, eggs, meats, cereals, and leaves distributed in three main meals and occasional enrichment programmes. Water was available ad libitum throughout the day. They voluntarily participated in the study and were never food or water deprived. Animal husbandry and research complied with the EAZA Minimum Standards for the Accommodation and Care of Animals in Zoos and Aquaria and the WAZA Ethical Guidelines for the Conduct of Research on Animals by Zoos and Aquariums, respectively. All apes were tested in testing rooms located at WKPRC.

Apparatus

The eye movements of ape participants were non-invasively recorded with an infrared eye-tracker (60 Hz; Tobii X120, Tobii Technology AB, Stockholm, Sweden) in an approximately $60-\mathrm{cm}$ viewing distance. We tested them unrestrained but separated from the experimenter and eye-tracker with a transparent acrylic panel. However, in order to keep their heads relatively still, we implemented a nozzle and tube attached to the acrylic panels, which produce grape juice little by little, and let the apes suck the nozzle during the recording (Figure 1a; also see Figure A1). No explicit training was conducted for the apes. Stimuli were presented on a 22-inch LCD monitor $(1366 \times 768$ pixel) with Tobii Studio software (version 3.2.1).

Two-point automated calibration was conducted for great apes by presenting a small object or video clip on each reference point. A relatively small number of reference points was adopted for apes because they tended to view those reference points only shortly. However, we manually checked the accuracy at five points after the initial 
calibration and repeated the calibration if necessary. As a result, our preliminary session confirmed the comparable accuracy between apes and humans (see Kano, Call, \& Tomonaga 2012 for the accuracy estimate). Before every test session for apes, we checked the accuracy manually and started the session when we confirmed that the error value was less than 1-2 degree.

\section{Stimuli and Procedure}

198 The stimuli were 10-second videos in which a model repeatedly looked at one of the two 199 identical objects (hereafter "target" as opposed to "distractor"). The model was either a male bonobo, chimpanzee, orangutan (one of the members from WKPRC), or a male human (F.K.) (Figure 1b). These models were familiar to the ape participants (the apes had at least some regular visual access even to allospecific models). Each model's head turn was videotaped at the testing room of WKPRC, and then later edited in Adobe Premire Pro so that the model appeared to look at the target repeatedly in the final video. The brightness and contrast were matched across stimuli as much as possible. The objects were plain coloured square shapes (the colour was selected not to stand out too much from the background; green for the bonobo video and red for the other videos, depending on the greenish/reddish background tones). In each video, the model faced

209 forward for 1 second, and for the remaining of time (9 s), repeatedly looked at the target by turning both eyes and head back and forth. The head turning frequency varied among stimuli in order to conserve the natural speed of each model's head turn $(4,5,5$,

2123 times respectively for bonobo, chimpanzee, human, and orangutan video; each head turn was thus about 1.8-3 s; see Video S1). 

was repeated for 3 times (total 6 trials). Each ape viewed a single video in a day (total 6 days). The order of presenting model type (conspecific or human) and direction of the model's gaze (left or right) was counterbalanced across individuals. The experimenter initiated the presentation of each video when apes were attending to the monitor.

Data analysis

Participants' fixations were detected by the Tobii fixation filter using Tobii Studio (version 3.2.1) with a default setting. To determine which areas each fixation landed on, we defined the area of interest (AOI) as a circle shape respectively for target, distractor (diameter 350 pixels), and model's face (diameter 400-700 pixels depending on the size of model's face; Figure 1c).

The main measurement was the proportion of trials in which the participants first looked at the target or distractor after the initiation of model's head turn (hereafter, the proportion of first look) with respect to total number of trials (including the trials in which participants looked at neither). We also measured the total number of fixations we report these results in detail in the Supplementary Material. In addition, to examine how rapidly each species responded to the gaze, we measured the response time when the participants first looked at the target (the initiation of looks at the target from the initiation of the model's head turn). Moreover, to examine whether the occurrence of gaze following was mediated by the strength of attention to the model's face, we measured the amount of time spent viewing for the 
model's face before the model's first head turn (hereafter, face viewing time) and correlated that value with the proportion of first look at the target. We standardized the face viewing time as the proportion of viewing time for face with respect to the total viewing time for the entire scene.

For the statistical analyses, we distinguished between within-species and between-species analyses. Within-species analysis tested whether each species show any evidence of gaze following and differential sensitivity to the conspecific versus human gaze (a repeated-measures ANOVA with Object and Model species as factors). Between-species analysis tested whether those species quantitatively differed from one another in their frequency of gaze following and their sensitivity to conspecific versus human gaze (a repeated-measures ANOVA with Subject species, Object, and Model species as factors). All analyses were conducted in SPSS (version 20).

Results

\section{Between-species analysis}

Figure 2 presents the means and standard errors of first look. A repeated-measures three-way ANOVA revealed a significant main effect of Object $\left(F_{1,26}=24.93, P<0.001\right.$, $\eta^{2}=0.49$ ). Thus, overall, great apes first looked at the target rather than the distractor more frequently than vice versa, indicating that they followed the model's gaze. There was a significant main effect of Subject species $\left(F_{2,26}=15.59, P=0.040, \eta^{2}=0.22\right)$. However, we also found a significant two-way interaction between Subject species and Object $\left(F_{2,26}=4.16, P=0.027, \eta^{2}=0.24\right)$. Thus, species also differed from one another in their frequency of first look at the target versus the distractor. More specifically, bonobos followed the gaze more frequently than chimpanzees $\left(F_{1,20}=16.74, P=0.001\right.$, 
$\left.262 \eta^{2}=0.45\right)$. Bonobos differed from chimpanzees particularly in their responses to the 263 human gaze $\left(F_{1,19}=7.22, P=0.015, \eta^{2}=0.27\right)$ rather than to the conspecific gaze $\left(F_{1,19}\right.$ $\left.264=1.62, P=0.21, \eta^{2}=0.07\right)$. Although bonobos and chimpanzees responded to conspecific versus human gaze somewhat differently, we did not find a significant three-way interaction between Model species, Subject species, and Object $\left(F_{2,26}=0.87\right.$, $\left.P=0.43, \eta^{2}=0.06\right)$. Orangutans did not significantly differ from either bonobos or chimpanzees in their frequency of first look at the target versus the distractor $(P s>0.1)$. We also examined the individual differences in terms of sex (male, female), age (young $\leqq 9$ year of age, adult $>9$ year of age), and rearing history (mother, hand reared) by including those factors into the same analysis. However, we did not find any significant effect of these factors ( $P S>0.1$ ), and importantly, the species difference in first look at the target versus the distractor was still detected in this follow-up analysis (Species $\times$ Object $; \quad F_{2,16}=3.96, P=0.040, \eta^{2}=0.33$ ).

We then examined whether the species difference in first look at the target was related to the species difference in face viewing time (how long they spent viewing the face before the model's first head turn). Species did not significantly differ from one another in their face viewing time $\left(F_{2,28}=2.62, P=0.092\right)$. In addition, there was no significant correlation between first look at the target and face viewing time (Pearson's $r$ $280=-0.12, N=29, P=0.51)$. Thus, it is unlikely that the species difference in overall frequency of gaze following was due to the variations of face viewing time. 
Within-species analysis

We then examined the pattern of first look respectively in each species using two-way ANOVAs. In bonobos, we found a significant main effect of Object $\left(F_{1,7}=19.38, P=\right.$ $\left.0.003, \eta^{2}=0.73\right)$ but not a significant interaction between Object and Model species $\left(F_{1}\right.$, $\left.{ }_{7}=0.038, P=0.85, \eta^{2}=0.005\right)$, indicating that they followed the gaze of both conspecific and human models. Similarly, in orangutans, we found a trend in the main effect of Object $\left(F_{1,6}=5.25, P=0.062, \eta^{2}=0.46\right)$ (we found a significant main effect of Object in the total number of fixations, see Supplementary Material) but not a significant interaction between Object and Model $\left(F_{1,6}=0.60, P=0.46, \eta^{2}=0.09\right)$. In chimpanzees, we did not find a significant main effect of Object $\left(F_{1,13}=1.63, P=0.22, \eta^{2}=0.11\right)$; yet we found a significant interaction between Object and Model $\left(F_{1,13}=5.06, P=0.042, \eta^{2}\right.$ 298 $=0.28$ ). Follow-up paired t-tests indicated that chimpanzees followed the gaze of conspecific model $\left(t_{13}=2.82, P=0.014\right)$ but not that of human model $\left(t_{13}=0.51, P=\right.$ $0.61)$.

We then examined whether chimpanzees' differential responses to the conspecific versus human gaze may be because of their differential viewing of the conspecific versus human face. As shown in Table 1, we indeed found that chimpanzees viewed the conspecific face longer than human face $\left(0.90\right.$ vs. $0.68 ; t_{13}=$ 3.87, $P=0.002)$. Moreover, as shown in Table 2, they viewed the face AOI longer on those trials in which they looked at the target (followed the gaze) than on those trials in which they looked at the distractor (0.88 vs. $\left.0.70 ; t_{8}=3.03, P=0.016\right)$. Thus, chimpanzees' preferential following of conspecific gaze may be simply explained by their preferential viewing of conspecific face. No such relation was confirmed for 
bonobos and orangutans ( $P s>0.07)$.

312 Response time

313 We examined the response time for first look (the time at which they first looked at the

314 target). We restricted this analysis to the presentation of conspecific models because

315 chimpanzees did not follow the human gaze. The response times were $1989 \pm 451$,

$3163364 \pm 359$, and $2621 \pm 501 \mathrm{~ms}$ (mean \pm S.E.), respectively for bonobos, chimpanzees,

317 and orangutans. Overall, there was some indication that species might differ in

318 response time $\left(F_{2,27}=2.85, P=0.076, \eta^{2}=0.41\right)$. Bonobos followed the conspecific

319 gaze significantly faster than chimpanzees $\left(t_{19}=2.37, P=0.028\right)$. Orangutans did not

320 differ from either bonobos or chimpanzees in their response time ( $P S>0.05)$.

321

Discussion

All species followed at least conspecific gaze in this eye-tracking paradigm. That is, they more frequently looked into the same than opposite direction with the conspecific model. As for the species difference, we found that bonobos followed human gaze more frequently than chimpanzees, which extends the similar finding in a previous study using a different behavioural paradigm (Herrmann, et al., 2010). Although the two species did not differ from one another in their frequency of following the conspecific gaze, bonobos followed the conspecific gaze faster than chimpanzees. Thus, bonobos seem to be more sensitive than chimpanzee to the gaze in general. Orangutans were not statistically different from the other two species in their frequency or timing of gaze following. 
334 (Hattori, et al., 2010), chimpanzees followed the conspecific gaze but not human gaze.

335 This preferential following of conspecific gaze seems to be related to their preferential

336 viewing of conspecific face. In addition, as shown in Figure 2, unlike chimpanzees,

337 bonobos frequently followed the gaze of both conspecific and human model. Thus, the

338 two species may differ from one another in their responses to conspecific versus

339 allospecific gaze. However, in Experiment 1, the statistical support for this idea was

340 insufficient (i.e. the three-way interaction; Model species $\times$ Subject species $\times$ Object).

341 Also, it remains unclear whether each species prefer not to follow only human gaze or

342 allospecific gaze in general. Also, for orangutans, although we did not find a statistical

343 difference between their responses to conspecific and allospecific, we also did not find a

344 clear statistical support for gaze following in response to the human gaze (see Figure 2).

345 Thus, we further explored great apes' responses to the allospecific gaze by presenting

346 the nonhuman allospecific gaze to the same participants in Experiment 3. 


\section{Experiment 2}

351 This experiment examined how infants and adults differently follow the gaze of human

352 and ape model (chimpanzee and orangutan model) with the same method used in

353 Experiment 1. Based on a previous study using an eye-tracking paradigm (Hattori, et al., 2010), we expected that human adults follow the gaze of both human and ape models. Although numerous studies have used nonhuman agents as stimuli to examine infants' social cognition in general, to the best of our knowledge, there is no previous study used nonhuman primates as stimuli to examine infants' gaze following. Thus, two different predictions are possible. One could hypothesize that human infants may follow both human and nonhuman gaze because previous studies have shown that human infants find goal-directedness in nonhuman agents if the agents show certain type of behavioural cues (e.g. self-propelling; Gergely \& Csibra 2003). However, some studies also suggest that human infants do not follow the gaze of nonhuman agents if the behavioural cues are limited. For example, infants followed the gaze of a toy animal only when the animal showed contingent movements to the infants prior to gaze cueing (Johnson, Slaughter, \& Carey, 1998). Also, the previous studies suggest that human infants, unlike great apes (Tomasello, Hare, Lehmann, \& Call, 2007), rely more on the eye than head direction when following the human gaze (Brooks \& Meltzoff, 2002). In this study, however, our ape models in the videos did not produce these cues explicitly. Critically, our ape models did not provide a clear signal of eye direction, unlike our human model, due to the nature of their eye morphology (i.e. dark sclera; Kobayashi \& Kohshima 1997). Importantly, in this experiment, we made some minor changes in our video stimuli. In our pilot test using the stimuli without any changes from Experiment 1, we 
found that human infants did not view the target/distractor object and instead kept looking at the face of human and ape model (while human adults followed the gaze). This is probably because infants generally move their gaze less frequently (i.e. sticky fixations) than great apes or human adults. Thus, based on the previous knowledge (Moore, 2008), in this experiment we made two minor changes to the stimuli used in Experiment 1 so that infants could release attention from the model's face and follow at least the gaze of human model (for details, see Method and Supplementary Results) as in many previous studies with the eye-tracking method (Senju \& Csibra, 2008; von Hofsten, et al., 2005).

\section{Method}

\section{Participants}

386 Twenty-two 12-month-old infants (within two weeks on either side; 11 males, 11 females) participated. The participants were recruited by telephone from a database of parents who had volunteered to participate in developmental studies. All parents agreed the informed consent upon coming to the institute. They were tested in a testing room located at the Max-Planck Institute for Evolutionary Anthropology (MPI-EVA), Leipzig, Germany. Two additional infants were tested but excluded from the analysis because of fussiness $(n=1)$ and a software malfunction $(n=1)$. We also asked one of the parents of

393 infants to participate in this study, and so 22 adults ( 7 males, 15 females, 20-40 years

394 old) completed the same trials as the infants.

\section{Apparatus}

396 The human participants were tested using the same eye-tracker, monitor, and software. Infants were seated on a parent's lap during the recording. Calibration was conducted 
using five reference points for infants and adults by presenting a small video at each reference point.

Stimuli and Procedure

401 Compared to the stimuli used in Experiment 1, we (1) increased the object saliency by 402 replacing them with colourful balls and (2) decreased the face saliency by reducing the 403 number of head turns of the model (only 2 times; see Video S2). Each infant and adult 404 viewed the videos of (1) human model and (2) ape species model. Half of the human 405 participants (11 infants and 11 adults) viewed the human and chimpanzee model and 406 the other half viewed the human and orangutan model. Each video was repeated 3 407 times (total 6 trials). Each infant and adult viewed all videos in a single session. The whole session lasted approximately 10 minutes. The presentation order for model type (conspecific or human) and direction of the model's gaze (left or right) was counterbalanced across individuals. At the beginning of each video, we presented small animations and boing sounds to make sure that infants looked at the monitor. Human

412 adults were told to watch the videos as they normally would. They were told neither the 413 contents of videos nor the purpose of experiments (i.e. gaze following) before

414 participating this experiment except that they would see apes and humans in the videos.

\section{Data analysis}

416 All analyses were conducted in the same way as in Experiment 1. Our initial analysis did 417 not reveal any significant difference between the two groups who saw the chimpanzee or orangutan model, and thus we combined the two groups in the following analyses.

420 Results

421 Proportion of first look 
Figure 3 presents the means and standard errors of first look. A repeated-measures three-way ANOVA revealed a significant main effect of Object $\left(F_{1,42}=53.92, P<0.001\right.$, $\eta^{2}=0.56$ ), indicating that they followed the gaze of models. There was a significant main effect of Age $\left(F_{1,42}=7.0, P=0.011, \eta^{2}=0.14\right)$, but also a significant interaction between Age and Object $\left(F_{1,42}=13.48, P=0.001, \eta^{2}=0.24\right)$. Thus, adults followed the gaze more frequently than infants. We also found a marginal three-way interaction between Model, Object, and Age $\left(F_{1,42}=3.45, P=0.070, \eta^{2}=0.076\right)$, suggesting that adults and infants followed the gaze of human and ape models differently.

We then examined the pattern of first look respectively in each age group using two-way ANOVAs. In adults, we found a significant main effect of Object $\left(F_{1,21}=53.76\right.$, $\left.P<0.001, \eta^{2}=0.71\right)$, but not the significant interaction between Model and Object $\left(F_{1,21}\right.$ $\left.=0.068, P=0.79, \eta^{2}=0.003\right)$. Thus, adults followed the gaze of both human and ape models. In infants, we found a significant main effect of Object $\left(F_{1,21}=7.73, P=0.011\right.$, $\left.\eta^{2}=0.26\right)$ but also a significant interaction between Model and Object $\left(F_{1,21}=11.29, P=\right.$ $\left.0.003, \eta^{2}=0.35\right)$. Follow-up paired t-tests for infants showed that they followed the human gaze $\left(t_{21}=3.83, P=0.001\right)$ but not the ape gaze $\left(t_{21}=0.86, P=0.39\right)$.

We also examined whether the age difference in first look was related to the face viewing time (how long they spent viewing the face before the model's first head turn). Adults viewed the model's face significantly longer than infants $\left(t_{42}=5.38, P<\right.$ 0.001). In addition, there was a significant correlation between first look at the target and face viewing time (Pearson's $r=0.39, N=44, P=0.007$ ). However, when we analysed each age group separately, we did not find any significant correlation in each group ( $P S$ $>0.5)$. Therefore, although adults followed the gaze more frequently and viewed the face longer than infants; the individual difference in face viewing time does not 
necessarily explain the individual difference in the first look.

Finally, we examined whether infants' differential responses to the human versus ape gaze may be because of their differential viewing of the human versus ape face. As shown in Table 1, we indeed found that infants viewed the human face longer than the ape face $\left(0.87\right.$ vs. $\left.0.64 ; t_{21}=3.41, P=0.003\right)$. Moreover, as shown in Table 2, they viewed the face $\mathrm{AOI}$ longer on those trials in which they looked at the target (followed the gaze) than on those trials in which they looked at the distractor (0.84 vs. $\left.0.75 ; t_{18}=3.45, P=0.003\right)$. Thus, infants' preferential following of human gaze may be mediated by their preferential viewing of human face. We did not conduct the same analysis for adults because they viewed both conspecific and allospecific faces over $90 \%$ of total time and rarely fixated on the distractor

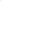

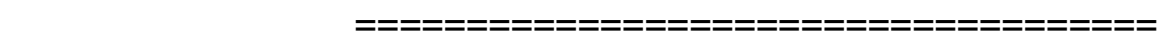

Figure 3 around here

\section{Response time}

As in Experiment 1, we restricted the analysis for response time to the presentation of human models because infants did not follow the gaze of ape models. The response times were $2518 \pm 276$ and $1516 \pm 231 \mathrm{~ms}$ (mean \pm S.E.), respectively for infants and adults. Adults follow the gaze significantly faster than infants $\left(t_{42}=2.78, P=0.008\right)$.

\section{Discussion}


Both human infants and adults followed at least the human model in this eye-tracking paradigm, consistent with many previous studies. Human adults followed the gaze more sensitively (more frequently and faster) than infants. Unlike adults, infants followed the conspecific (human) but not ape gaze. This preferential following of conspecific face by infants seems to be related to their preferential viewing of human face. . It is not surprising to find that human adults follow both human and allospecific ape gaze more sensitively than infants, given their sensitivity to both human and allospecific ape eyes (Kano \& Tomonaga, 2010) and a strong contagious tendency toward the other's behaviours (Driver et al., 1999; Gallup et al., 2012). Also, human adults are usually given numerous opportunities to view humans and nonhumans in media and to interact with them in a real life. On the other hand, it is somewhat surprising to find that human infants showed marked differences in their responses to conspecific versus allospecific faces given that human infants attribute goal-directedness of nonhuman agents in many contexts (Gergely \& Csibra, 2003). Infants' preferential gaze following was analogous to chimpanzees'. We will discuss the 484 possible mechanism underlying this apparent similarity between human infants and chimpanzees in the General Discussion. 


\section{Experiment 3}

489 This experiment further investigated whether great apes followed the allospecific gaze;

490 yet this time, we used other ape species as models. The purpose of this experiment was

491 to complement the crossed design with two factors, Subject species and Model species.

492 In particular, in Experiment 1, we found (1) chimpanzees' conspecific preference and (2)

493 bonobos' gaze sensitivity over chimpanzees'. However, since humans are very familiar

494 allospecifics to ape participants (e.g. caregivers), it is not entirely clear whether

495 Experiment 1's findings derive from their special response to the human model or from

496 general response to the allospecific model. If the latter were the case, the same pattern

497 of results of Experiment 1 would emerge also in this experiment.

498 Method

499 We tested the same ape participants using the same stimuli as in Experiment 1, but

500 presenting the chimpanzee and bonobo models to the bonobo and chimpanzee 501 participants, respectively. We also presented the bonobo model to the orangutan 502 participants (because the orangutan participants at the WKPRC had a better visual 503 access to the bonobo model than the chimpanzee model used in this study). The 504 presentation order of direction of the model's gaze (left or right) was counterbalanced 505 across individuals. The apparatus and other procedures are the same as in Experiment $506 \quad 1$

507 Results and Discussion

508 Figure 4 presents the means and standard errors of first look. A repeated-measures 509 two-way ANOVA revealed a significant main effect of Object $\left(F_{1,26}=24.31, P=0.012, \eta^{2}\right.$ $510=0.28)$ but also a significant interaction between Object and Subject species $\left(F_{2,26}=\right.$ $\left.5115.29, P<0.001, \eta^{2}=0.48\right)$. Specifically, bonobos followed the gaze of chimpanzee 
512 model $\left(t_{7}=3.45, P=0.011\right)$, and orangutans followed the gaze of bonobo model $\left(t_{6}=\right.$

$5136.0, P=0.001)$. In contrast, chimpanzees did not follow the gaze of bonobo models $\left(t_{13}\right.$

$514=0.51, P=0.61)$. We omitted the correlation analysis with the first look and face viewing

515 time in this experiment due to the insufficient number of trials. The response times for

516 the first look at the target were $2165 \pm 463$ and $3469 \pm 715$ (mean \pm S.E.), respectively

517 for bonobos and orangutans (not significantly different, $P>0.1$ ).

$518 \quad$ Thus, taken together with the results from Experiment 1, chimpanzees seem to

519 differ from the other two species in their responses to the allospecific gaze. That is,

520 while bonobos and orangutans followed the gaze of allospecific models, chimpanzees

521 did not follow the gaze of allospecific models, either human or allospecific ape (bonobo)

522 model.

$523 \quad$ Finally, it should be noted that, although this study (and the previous study)

524 showed that chimpanzees followed the conspecific gaze, further studies are necessary

525 to pin down what type of conspecific models they prefer to follow. That is, this study (and

526 the previous study) used a familiar chimpanzee as a model, and thus it is possible that

527 their preferential gaze following may reflect their preference for familiar individuals (or

528 in-group individuals) rather than their preference for conspecific individuals in general.

529 This issue will be further discussed in General Discussion.

530 
537 In this study, we found that all species similarly followed the gaze of conspecific model.

538 However, while bonobos, orangutans, and human adults followed the gaze of both 539 conspecific and allospecific models, chimpanzees and human infants followed the gaze 540 of only conspecific models. Importantly, all stimulus models elicited the gaze following 541 responses in at least two species, and overall patterns for the presence/absence of 542 gaze following were unrelated to the low-level stimulus differences (e.g. colour, 543 brightness, and contract of objects/models; but note some changes in the stimuli in 544 Experiment 2). Rather, the absence of following the allospecific gaze by chimpanzees and human infants was related to their inattentiveness to the allospecific face.. This seems to indicate that motivational differences of participants, not the competence differences of participants nor the low-level differences of stimuli, are responsible for the observed patterns of gaze following in these species.

Preferential following of conspecific gaze by chimpanzees and infants suggests that they may preferentially learn from or acquire information from the conspecifics, the most relevant others. That is, rather than reflexively following any individual's gaze, they may first selectively view the relevant others and then follow the gaze. This behavioural strategy, "select-then-follow", may be particularly important for those species like chimpanzees and young humans who need to learn efficiently from particular individuals.. Thus, at least in this sense, this study is consistent with the previous studies reporting selective social referencing or selective behavioural copying of particular others, such as majorities and dominants, by chimpanzees, capuchin monkeys, vervet monkeys, and human children (Dindo, Whiten, \& de Waal, 2009; Haun, Rekers, \& Tomasello, 2012; van de Waal, Renevey, Favre, \& Bshary, 2010). 
However, our findings about conspecific preference by chimpanzees and

human infants are limited in scope because it is unclear whether they followed the gaze of conspecifics in general or only that of particular individuals (e.g. familiar/unfamiliar individuals). Since previous studies have shown that the familiarity of the model modulates the gaze following in monkeys and human infants and adults (Deaner, Shepherd, \& Platt, 2007; Gredebäck, Fikke, \& Melinder, 2010; Micheletta \& Waller, 2012), it is possible that our human and ape participants have some specific preference for particular individuals. This is an issue that deserves further investigation especially given that several studies have shown that communicative signals of strangers may function differently for human infants and nonhuman animals (Topál, Gergely, Erdőhegyi, Csibra, \& Miklósi, 2009).

In this study, we observed particularly intriguing species differences between bonobos and chimpanzees. First, unlike chimpanzees, bonobos did not show preference in following the conspecific versus allospecific gaze. Thus, bonobos followed the allospecific gaze more frequently than chimpanzees. Second, although the two species did not significantly differ from one another in their frequency of following the conspecific gaze, bonobos followed the conspecific gaze significantly faster than chimpanzees. These species differences in gaze following seem to be unrelated to their attentiveness to the model's face. Taken together, bonobos seem to differ from chimpanzees in their intrinsic sensitivity to the gaze. One proximate explanation for this species difference is that bonobos may follow the gaze more reflexively and thus less selectively than chimpanzees. In support of this view, a previous study based on a behavioural paradigm showed that bonobos and chimpanzees followed the experimenter's gaze but chimpanzees inferred the location of hidden objects more 
flexibly (thus perhaps less reflexively) in various experimental contexts than bonobos

585 (MacLean \& Hare, 2012). As for the underlying mechanism, several previous studies with macaques and humans have suggested that the relative strength of reflexive and voluntary components in gaze following may be modulated by androgen-related mechanisms (Shepherd, et al., 2006). Previous studies have also shown that bonobos and chimpanzees are different in their levels or reactivity of androgens (Wobber et al., 2010). Thus, the physiological differences between species may contribute to how much reflectively or selectively each species follows gaze. To test this possibility, again future studies should examine to what extent bonobos and chimpanzees are selective in following the gaze of particular conspecific individuals.

The observed patterns of gaze following by orangutans fit somewhat in between bonobos and chimpanzees in terms of the frequency and selectiveness. Importantly, in this study, orangutans reliably followed the model's gaze, and in no case they were significantly inferior to the other two species. One of the main differences between this study and previous ones is that this study measured their eye- rather than head/body-movements. Thus, orangutans in this study should have been free of physical constraints derived from their relatively slow head/body movements. Also, this study used a straightforward experimental setting which only required orangutans to glance at the object existing in their visual fields. Thus, at least at the basic level, it is reasonable to conclude that orangutans do not differ from the other great ape species in their ability of gaze following. discussed above, this pattern resembles that of chimpanzees, and the function may be also similar between the two species. The underlying mechanisms may be also similar 

between chimpanzees and infants. That is, human infants may have tried to selectively acquire information from the human models as relevant others, thereby viewed the human face longer than ape face, and followed the human gaze more frequently than the ape gaze. However, based on the previous studies with human infants, alternative explanations are also possible. That is, first, acknowledging the model's communicative

613 intent, which is provided as such signal as eye contact and contingent movements, is 614 important to elicit gaze following in infants (Farroni, Csibra, Simion, \& Johnson, 2002; 615 Senju \& Csibra, 2008). Thus, our infants may have failed to see the communicative 616 intent in the ape models because they had little experience in seeing and interacting 617 with great apes or similar kinds. Second, human infants, but not great apes, are sensitive to the eye rather than head direction of human models (Brooks \& Meltzoff, 2002; Tomasello, Hare, Lehmann, \& Call, 2007). Thus, our infants may have failed to perceive the eye direction of ape models because the apes do not have a clear contrast between iris and sclera unlike humans (Kobayashi \& Kohshima, 1997). Neither of those accounts could be applied to our chimpanzees' preferential gaze following of conspecific chimpanzee models. Thus, it is possible that distinct mechanisms underlie 624 the apparently similar pattern of gaze following between chimpanzees and human 625 infants. Likewise, although bonobos' and human adults' sensitivity to the allospecific 626 gaze may reflect a similar mechanism to some extent, e.g. reflexive following of any gaze, it is also possible that distinct mechanisms underlie the apparent similarities, especially given human adults' extensive experience with allospecific faces. allospecific gaze suggests the importance of using conspecific models in the comparative studies of gaze following and perhaps any social behaviours relying on 
632 gaze following (also see Hare, et al., 2000; Tomasello, et al., 1998). However, it should

633 be noted that, although chimpanzees in this study did not follow the human gaze,

634 numerous previous studies have documented their robust responses to the human 635 experimenter's gaze. Parsimoniously, this disparity can be explained by the 636 methodological differences; in this study chimpanzees spontaneously pay less attention 637 to the human face than the conspecific face, but in the previous studies, the human 638 experimenter typically establishes the eye contact with chimpanzees before giving a 639 gaze cue (by presenting a food in front of the face or calling the chimpanzee's name). 640 Also, the relevance of stimuli (i.e. video versus live) may also contribute to the 641 chimpanzees' motivation of attending to the human face.

642

643 Conclusion

644 Using a crossed design with two factors, Subject species and Model species, we 645 showed that (1) all species followed the conspecific gaze, (2) unlike bonobos, 646 orangutans and human adults, chimpanzees and human infants preferentially followed 647 the conspecific but not allospecific gaze, and (3) bonobos followed both conspecific and 648 allospecific gaze more sensitively than chimpanzees. Thus, we conclude that gaze 649 following is modulated by both Subject species and Model species in great apes and 650 humans, presumably a reflection of the subjects' intrinsic sensitivity to gaze and also 651 their selective interest in particular models.

652 
654

655

656

657

658

659

660

661

662

663

664

665

666

667

668

669

670

671

672

673

674

675

676

677

References

Amici, F., Aureli, F., Visalberghi, E., \& Call, J. (2009). Spider monkeys (Ateles geoffroyı) and capuchin monkeys (Cebus apella) follow gaze around barriers: Evidence for perspective taking? Journal of Comparative Psychology, 123(4), 368-374.

Anderson, J. R., \& Mitchell, R. W. (1999). Macaques but not lemurs co-orient visually with humans. Folia Primatologica, 70(1), 17-22.

Bräuer, J., Call, J., \& Tomasello, M. (2005). All great ape species follow gaze to distant locations and around barriers. Journal of Comparative Psychology, 119(2), 145-154.

Brooks, R., \& Meltzoff, A. N. (2002). The importance of eyes: how infants interpret adult looking behavior. Developmental Psychology, 38(6), 958-966.

Bugnyar, T., Stöwe, M., \& Heinrich, B. (2004). Ravens, Corvus corax, follow gaze direction of humans around obstacles. Proceedings of the Royal Society of London. Series B: Biological Sciences, 271(1546), 1331-1336.

Burkart, J., \& Heschl, A. (2006). Geometrical gaze following in common marmosets (Callithrix jacchus). Journal of Comparative Psychology, 120(2), 120-130.

Chow, V., Poulin - Dubois, D., \& Lewis, J. (2008). To see or not to see: Infants prefer to follow the gaze of a reliable looker. Developmental Science, 11(5), 761-770.

Corkum, V., \& Moore, C. (1998). The origins of joint visual attention in infants. Developmental Psychology, 34(1), 28-38.

D'Entremont, B., Hains, S., \& Muir, D. (1997). A demonstration of gaze following in 3-to 6-month-olds. Infant Behavior and Development, 20(4), 569-572.

Deaner, R. O., Shepherd, S. V., \& Platt, M. L. (2007). Familiarity accentuates gaze cuing in women but not men. Biology Letters, 3(1), 64-67. 
Dindo, M., Whiten, A., \& de Waal, F. B. (2009). In-group conformity sustains different foraging traditions in capuchin monkeys (Cebus apella). PLoS One, 4(11), e7858.

Driver, J., Davis, G., Ricciardelli, P., Kidd, P., Maxwell, E., \& Baron-Cohen, S. (1999). Gaze perception triggers reflexive visuospatial orienting. Visual cognition, 6(5), 509-540.

Emery, N. J., Lorincz, E. N., Perrett, D. I., Oram, M. W., \& Baker, C. I. (1997). Gaze Following and Joint Attention in Rhesus Monkeys (Macaca mulatta). Journal of Comparative Psychology, 111, 286-293.

Farroni, T., Csibra, G., Simion, F., \& Johnson, M. H. (2002). Eye contact detection in humans from birth. Proceedings of the National Academy of Sciences, 99(14), 9602.

Gallup, A. C., Hale, J. J., Sumpter, D. J., Garnier, S., Kacelnik, A., Krebs, J. R., et al. (2012). Visual attention and the acquisition of information in human crowds. Proceedings of the National Academy of Sciences, 109(19), 7245-7250.

Gergely, G., \& Csibra, G. (2003). Teleological reasoning in infancy: The naıve theory of rational action. Trends in Cognitive Sciences, 7(7), 287-292.

Goossens, B., Dekleva, M., Reader, S. M., Sterck, E. H., \& Bolhuis, J. J. (2008). Gaze following in monkeys is modulated by observed facial expressions. Animal Behaviour, 75(5), 1673-1681.

Gredebäck, G., Fikke, L., \& Melinder, A. (2010). The development of joint visual attention: a longitudinal study of gaze following during interactions with mothers and strangers. Developmental Science, 13(6), 839-848.

Hare, B., Call, J., \& Tomasello, M. (2000). Chimpanzees know what conspecifics do and 
do not see. Animal Behaviour, 59, 771-785.

Hattori, Y., Kano, F., \& Tomonaga, M. (2010). Differential sensitivity to conspecific and allospecific cues in chimpanzees and humans: A comparative eye-tracking study. Biology Letters, 6(5), 610-613.

Haun, D., Rekers, Y., \& Tomasello, M. (2012). Majority-biased transmission in chimpanzees and human children, but not orangutans. Current Biology, 22(8), 727-731.

Herrmann, E., Call, J., Hernandez-Lloreda, M. V., Hare, B., \& Tomasello, M. (2007). Humans have evolved specialized skills of social cognition: The cultural intelligence hypothesis. Science, 317(5843), 1360-1366.

Herrmann, E., Hare, B., Call, J., \& Tomasello, M. (2010). Differences in the cognitive skills of bonobos and chimpanzees. PLoS One, 5(8), e12438.

Hood, B. M., \& Atkinson, J. (1993). Disengaging visual attention in the infant and adult. Infant Behavior and Development, 16(4), 405-422.

Hood, B. M., Willen, J. D., \& Driver, J. (1998). Adult's eyes trigger shifts of visual attention in human infants. Psychological Science, 9(2), 131-134.

Itakura, S., Agnetta, B., Hare, B., \& Tomasello, M. (1999). Chimpanzee use of human and conspecific social cues to locate hidden food. Developmental Science, 2(4), 448-456.

Johnson, S., Slaughter, V., \& Carey, S. (1998). Whose gaze will infants follow? The elicitation of gaze-following in 12-month-olds. Developmental Science, 1(2), 233-238.

Kaminski, J., Riedel, J., Call, J., \& Tomasello, M. (2005). Domestic goats, Capra hircus, follow gaze direction and use social cues in an object choice task. Animal 

Behaviour, 69(1), 11-18.

727 Kano, F., Call, J., \& Tomonaga, M. (2012). Face and eye scanning in gorillas, orangutans, and humans: Unique eye-viewing patterns in humans among hominids. Journal of Comparative Psychology, 126(4), 388-398.

Kano, F., Hirata, S., Call, J., \& Tomonaga, M. (2011). The visual strategy specific to humans among hominids: A study using the gap-overlap paradigm. Vision Research, 51(23), 2348-2355.

Kano, F., \& Tomonaga, M. (2010). Face scanning in chimpanzees and humans: Continuity and discontinuity. Animal Behaviour, 79(1), 227-235.

Kaplan, G., \& Rogers, L. J. (2002). Patterns of gazing in orangutans (Pongo pygmaeus). International Journal of Primatology, 23(3), 501-526.

Kobayashi, H., \& Kohshima, S. (1997). Unique morphology of the human eye. Nature, 387(6635), 767-768.

Loretto, M.-C., Schloegl, C., \& Bugnyar, T. (2010). Northern bald ibises follow others' gaze into distant space but not behind barriers. Biology Letters, 6(1), 14-17.

MacLean, E. L., \& Hare, B. (2012). Bonobos and chimpanzees infer the target of another's attention. Animal Behaviour, 83(2), 345-353.

Micheletta, J., \& Waller, B. M. (2012). Friendship affects gaze following in a tolerant species of macaque (Macaca nigra). Animal Behaviour, 83(2), 459-467.

Moll, H., \& Tomasello, M. (2004). 12- and 18-month-old infants follow gaze to spaces behind barriers. Developmental Science, 7(1), 1-9.

Moore, C. (2008). The development of gaze following. Child Development Perspectives, 2(2), 66-70.

Okamoto-Barth, S., Call, J., \& Tomasello, M. (2007). Great apes' understanding of other 

individuals' line of sight. Psychological Science, 18(5), 462-468.

751

Rosati, A. G., \& Hare, B. (2009). Looking past the model species: diversity in gaze-following skills across primates. Current Opinion in Neurobiology, 19(1), $45-51$.

Ruiz, A., Gómez, J. C., Roeder, J. J., \& Byrne, R. W. (2009). Gaze following and gaze priming in lemurs. Animal Cognition, 12(3), 427-434.

Sandel, A. A., MacLean, E. L., \& Hare, B. (2011). Evidence from four lemur species that ringtailed lemur social cognition converges with that of haplorhine primates. Animal Behaviour, 81(5), 925-931.

Scerif, G., Gomez, J.-C., \& Byrne, R. W. (2004). What do Diana monkeys know about the focus of attention of a conspecific? Animal Behaviour, 68(6), 1239-1247.

Senju, A., \& Csibra, G. (2008). Gaze following in human infants depends on communicative signals. Current Biology, 18(9), 668-671.

Shepherd, S. V., Deaner, R. O., \& Platt, M. L. (2006). Social status gates social attention in monkeys. Current Biology, 16(4), 119-120.

Shepherd, S. V., \& Platt, M. L. (2008). Spontaneous social orienting and gaze following in ringtailed lemurs (Lemur catta). Animal Cognition, 11(1), 13-20.

Téglás, E., Gergely, A., Kupán, K., Miklósi, Á., \& Topál, J. (2012). Dogs' gaze following is tuned to human communicative signals. Current Biology, 22(3), 1-4.

Teufel, C., Gutmann, A., Pirow, R., \& Fischer, J. (2010). Facial expressions modulate the ontogenetic trajectory of gaze-following among monkeys. Developmental Science, 13(6), 913-922.

Tomasello, M., Call, J., \& Hare, B. (1998). Five primate species follow the visual gaze of conspecifics. Animal Behaviour, 55(4), 1063-1069. 
774 Tomasello, M., Hare, B., \& Agnetta, B. (1999). Chimpanzees, Pan troglodytes, follow gaze direction geometrically. Animal Behaviour, 58(4), 769-777.

776 Tomasello, M., Hare, B., Lehmann, H., \& Call, J. (2007). Reliance on head versus eyes in the gaze following of great apes and human infants: The cooperative eye hypothesis. Journal of Human Evolution, 52(3), 314-320.

Topál, J., Gergely, G., Erdőhegyi, Á., Csibra, G., \& Miklósi, Á. (2009). Differential sensitivity to human communication in dogs, wolves, and human infants. Science, 325(5945), 1269-1272.

van de Waal, E., Renevey, N., Favre, C. M., \& Bshary, R. (2010). Selective attention to 783 philopatric models causes directed social learning in wild vervet monkeys. Proceedings of the Royal Society B: Biological Sciences, 277(1691), 2105-2111. von Hofsten, C., Dahlstrom, E., \& Fredriksson, Y. (2005). 12-Month-Old Infants' Perception of Attention Direction in Static Video Images. Infancy, 8(3), 217-231.

Wilkinson, A., Mandl, I., Bugnyar, T., \& Huber, L. (2010). Gaze following in the red-footed tortoise (Geochelone carbonaria). Animal Cognition, 13(5), 765-769.

Wobber, V., Hare, B., Maboto, J., Lipson, S., Wrangham, R., \& Ellison, P. T. (2010). Differential changes in steroid hormones before competition in bonobos and chimpanzees. Proceedings of the National Academy of Sciences, 107(28), 12457-12462.

793

794 
Appendix

\section{Total number of fixations}

798 Overall, we obtained similar results with this measure as compared to the proportion of

799 first look.

\section{Experiment 1}

801 Figure A2 presents the means and standard errors of total number of fixations. A 802 repeated-measures three-way ANOVA (Object, Subject species, Model species) 803 revealed a significant main effect of Object $\left(F_{1,26}=6.77, P<0.001, \eta^{2}=0.41\right)$ and 804 Subject species $\left(F_{2,26}=9.82, P=0.001, \eta^{2}=0.43\right)$ and a significant interaction between 805 Subject species and Object $\left(F_{2,26}=6.97, P=0.004, \eta^{2}=0.34\right)$. Bonobos and chimpanzees differ from one another in their total number of fixations on the target versus the distractor $\left(F_{1,20}=11.77, P=0.003, \eta^{2}=0.37\right)$. Orangutans did not differ from the other two species $(P>0.1)$. Within-species analysis revealed a significant main effect of Object for bonobos $\left(F_{1,7}=8.58, P=0.022, \eta^{2}=0.55\right)$ and for orangutans $\left(F_{1,6}\right.$

$\left.810=8.09, P=0.029, \eta^{2}=0.57\right)$, but not for chimpanzees $\left(F_{1,13}=0.21, P=0.65, \eta^{2}=0.01\right)$.

811 There was a significant interaction between Object and Model for chimpanzees $\left(F_{1,13}=\right.$ $\left.8125.41, P=0.037, \eta^{2}=0.29\right)$ but not for the other two species $(P s>0.5)$.

\section{Experiment 2}

814 Figure $\mathrm{A} 3$ presents the means and standard errors of total number of fixations. A repeated-measures three-way ANOVA (Object, Age, Model species) revealed a significant main effect of Object $\left(F_{1,42}=147.88, P<0.001, \eta^{2}=0.59\right)$ and Age $\left(F_{1,42}=\right.$ 4.33, $\left.P=0.044, \eta^{2}=0.09\right)$ and a significant interaction between Object and Age $\left(F_{1,42}=\right.$ 19.62, $\left.P<0.001, \eta^{2}=0.31\right)$. When we analysed each group separately, in adults, we

819 found a significant main effect of Object $\left(F_{1,21}=40.88, P<0.001, \eta^{2}=0.66\right)$ but not a 
significant interaction between Model and Object $\left(F_{1,21}=1.91, P=0.18, \eta^{2}=0.08\right)$. In

821 infants, we found a significant main effect of Object $\left(F_{1,21}=40.88, P<0.001, \eta^{2}=0.66\right)$

822 but also a significant interaction between Model and Object $\left(F_{1,21}=10.69, P=0.004, \eta^{2}\right.$

$823=0.33)$.

\section{Experiment 3}

825 Figure A4 presents the means and standard errors of total number of fixations. A 826 repeated-measures two-way ANOVA revealed a significant main effect of Object $\left(F_{1,26}=\right.$ $\left.82715.84, P<0.001, \eta^{2}=0.37\right)$. We did not find a significant interaction between Object and 828 Subject species $\left(F_{2,26}=2.28, P=0.12, \eta^{2}=0.14\right)$. When we analysed each species separately, we found a significant effect of Object for orangutans $\left(t_{6}=4.76, P=0.003\right)$, a trend for bonobos $\left(t_{7}=2.29, P=0.056\right)$, and no significant effect for chimpanzees $\left(t_{13}=\right.$ $0.88, P=0.39)$.

832

\section{The pilot test for Experiment 2 (infants and adults)}

834 A pilot test was conducted for Experiment 2 with a separate group of infants and adults

835 using the same stimuli as in Experiment 1.

\section{Method}

837 Eleven infants ( 6 males, 7 females) and 11 adults ( 3 males, 8 females) participated in 838 this pilot test. They were presented with the videos of chimpanzee and human model, 839 which were the same as those used in Experiment 1. All the other procedures were 840 same as those in the main test (Experiment 2).

841 Results

842 As shown in Figure A5 and A6, although control adults followed the gaze of both models 843 ( $P s<0.01)$, infants did not follow the gaze of either $(P s>0.2)$. In most of the trials, 
844 infants did not view the objects but instead kept viewing the faces of models.

845 Discussion

846 Such "sticky" fixations of infants to the faces may derive from their immaturity of 847 attention (Hood, Willen, \& Driver, 1998). In particular, the objects in our stimuli may be

848 too simple in the forms (plain colored squares), and also the faces of models may be too

849 attractive in the motions (frequent head turns) to release their attention from faces. We

850 therefore changed those parameters In Experiment 2 and improved their performance 851 (see the main text).

852 One might expect that, by applying the same changes, great apes would also

853 show improved gaze-following performances. However, we doubt this possibility 854 because apes fixated the face far more briefly than do any humans (in this experiment 855 and also in general; Kano, Call, \& Tomonaga 2012).

856

857

858

859 
860 Tables

861

Table 1. Proportion of face viewing time (mean, S.E.) for each model species

\begin{tabular}{lcc}
\hline & \multicolumn{2}{c}{ Model species } \\
\cline { 2 - 3 } Subject species & Ape & Human \\
\hline Bonobos (Exp. 1) & $0.66(0.069)$ & $0.57(0.051)$ \\
Chimpanzees (Exp. 1) & $0.90(0.032)$ & $0.68(0.039)$ \\
Orangutans (Exp. 1) & $0.83(0.051)$ & $0.70(0.019)$ \\
Human infants (Exp.2) & $0.71(0.052)$ & $0.88(0.023)$ \\
\hline
\end{tabular}

862

Table 2. Proportion of face viewing time (mean, S.E.) as a function of the looking patterns for the objects.

\begin{tabular}{lccc}
\hline & \multicolumn{3}{c}{ Looking pattern } \\
\cline { 2 - 4 } Subject species & $\begin{array}{c}\text { Looked at } \\
\text { neither }\end{array}$ & $\begin{array}{c}\text { Looked at } \\
\text { target }\end{array}$ & $\begin{array}{c}\text { Looked at } \\
\text { distractor }\end{array}$ \\
\hline Bonobos (Exp. 1) & $0.60(0.14)$ & $0.62(0.057)$ & $0.62(0.059)$ \\
Chimpanzees (Exp. 1) & $0.80(0.054)$ & $0.88(0.030)$ & $0.70(0.045)$ \\
Orangutans (Exp. 1) & $0.79(0.031)$ & $0.75(0.049)$ & $0.78(0.071)$ \\
Human infants (Exp. & & & \\
2) & $0.78(0.060)$ & $0.84(0.029)$ & $0.76(0.044)$ \\
\hline
\end{tabular}

863 
Table A1. Species, sex, age, and rearing history of the ape subjects

\begin{tabular}{lllrl}
\hline Name & Species & Sex & Age & Rearing history \\
\hline Fimi & Bonobo & F & 5 & Mother \\
Luiza & Bonobo & F & 8 & Mother \\
Yasa & Bonobo & F & 15 & Mother \\
Ulindi & Bonobo & F & 19 & Mother \\
Loto & Bonobo & M & 3 & Mother \\
Kuno & Bonobo & M & 16 & Hand Reared \\
Jasongo & Bonobo & M & 23 & Mother \\
Joey & Bonobo & M & 30 & Hand Reared \\
Kara & Chimpanzee & F & 8 & Mother \\
Fifi & Chimpanzee & F & 20 & Mother \\
Jahaga & Chimpanzee & F & 20 & Mother \\
Sandra & Chimpanzee & F & 20 & Mother \\
Getrudia & Chimpanzee & F & 20 & Mother \\
Riet & Chimpanzee & F & 35 & Hand Reared \\
Ulla & Chimpanzee & F & 36 & Hand Reared \\
Fraukje & Chimpanzee & F & 37 & Hand Reared \\
Bangolo & Chimpanzee & M & 4 & Mother \\
Kofi & Chimpanzee & M & 8 & Mother \\
Lobo & Chimpanzee & M & 9 & Mother \\
Alex & Chimpanzee & M & 12 & Hand Reared \\
Lome & Chimpanzee & M & 12 & Mother \\
Robert & Chimpanzee & M & 37 & Hand Reared \\
Raja & Orangutan & F & 9 & Mother \\
Padana & Orangutan & F & 15 & Mother \\
Dokana & Orangutan & F & 24 & Mother \\
Pini & Orangutan & F & 25 & Mother \\
Batak & Orangutan & M & 3 & Mother \\
Suaq & Orangutan & M & 4 & Mother \\
Tanah & Orangutan & M & 4 & Mother \\
\hline
\end{tabular}


Figure legends

867 Figure 1: Overview of experiments. (a) The participants and (b) the models in this study.

868 (c) An example of area of interest (AOI) defined for the fixation analysis. In this study, 869 bonobos were presented with the bonobo, chimpanzee, and human models.

870 Chimpanzees were presented with the chimpanzee, bonobo, and human models.

871 Orangutans were presented with the orangutan, human, and bonobo models. Human

872 infants and adults were presented with the human, chimpanzee, and orangutan models.

873 Thus, each species was presented with three types of models, and each model was 874 presented to at least three species.

875 Figure 2: Proportion of first look in great apes when they were viewing conspecific and 876 human models. Error bars denote the standard error of mean. $+P<0.07,{ }^{*} P<0.05$, $87{ }^{* \star \star} P<0.001$.

Figure 3: Proportion of first look in human infants and adults when they were viewing human and ape models. Error bars denote the standard error of mean. ${ }^{* \star \star} P<0.001$.

880 Figure 4: Proportion of first look in great apes when they were viewing allospecific ape

881 models. Error bars denote the standard error of mean. ${ }^{\star} P<0.05,{ }^{* *} P<0.001$.

882 Figure A1: An ape on the apparatus (a) and the apparatuses in Experiment 1 (great 883 apes).

884 Figure A2: Total number of fixations in great apes when they were viewing conspecific 885 and human models. Error bars denote the standard error of mean. ${ }^{*} P<0.05$, ${ }^{* *} P<$ $886 \quad 0.001$.

Figure A3: Total number of fixations in human infants and adults when they were 
888 viewing human and ape models. Error bars denote the standard error of mean. ${ }^{\star \star \star} P<$ $889 \quad 0.001$.

890 Figure A4: Total number of fixations in great apes when they were viewing allospecific 891 ape models. Error bars denote the standard error of mean. $+P<0.07,{ }^{* *} P<0.01$

892 Figure A5: Proportion of first look in human infants and adults when they were viewing 893 human and ape models (Pilot test for Experiment 2). Error bars denote the standard 894 error of mean. ${ }^{* *} P<0.01,{ }^{*}:{ }^{*} P<0.001$

895 Figure A6: Total number of fixations in human infants and adults when they were 896 viewing human and ape models (Pilot test for Experiment 2). Error bars denote the 897 standard error of mean. ${ }^{* *} P<0.01,{ }^{*}:{ }^{*} P<0.001$

898 
(a)

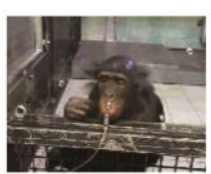

Bonobos $(N=8)$

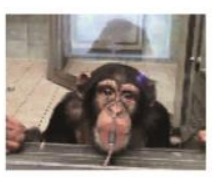

Chimpanzees $(N=14)$

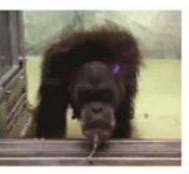

Orangutans $(N=7)$

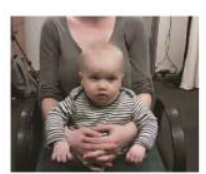

Human infants $(n=22)$ adults $(n=22)$

(b)

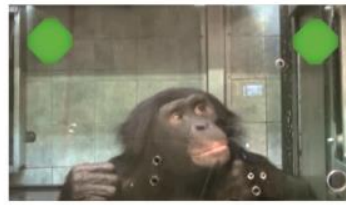

A bonobo model

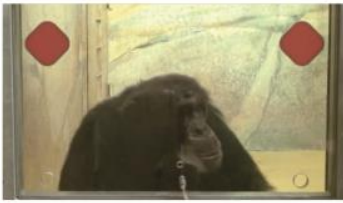

A chimpanzee model

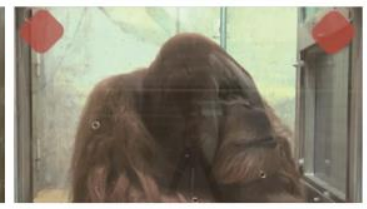

A orangutan model

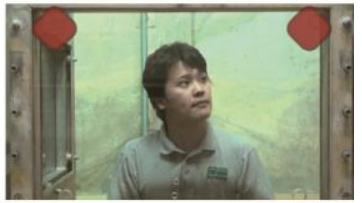

A human model

899

(c)

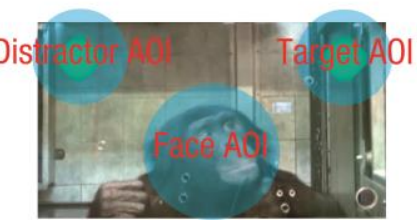

900 


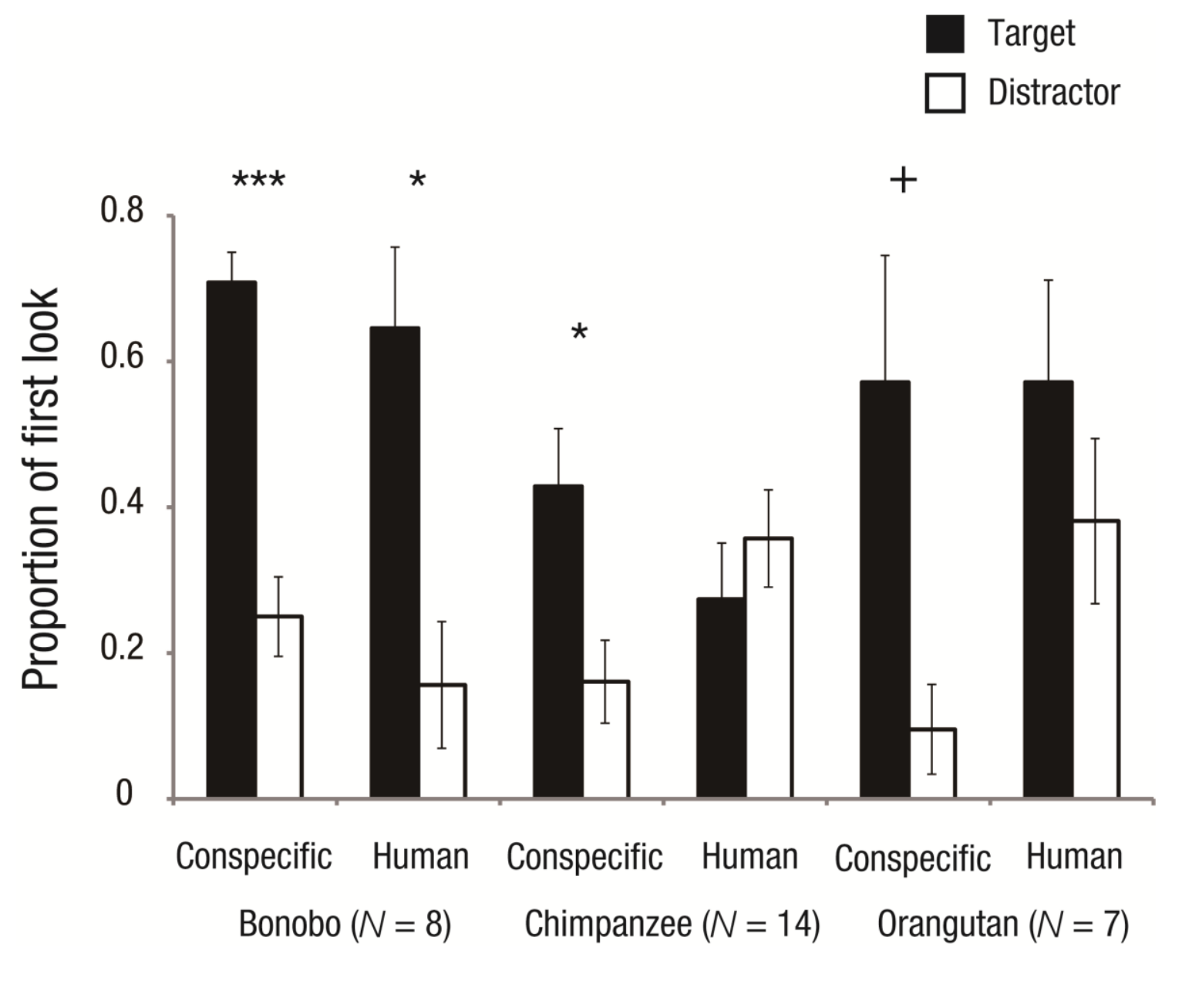


Target

$\square$ Distractor

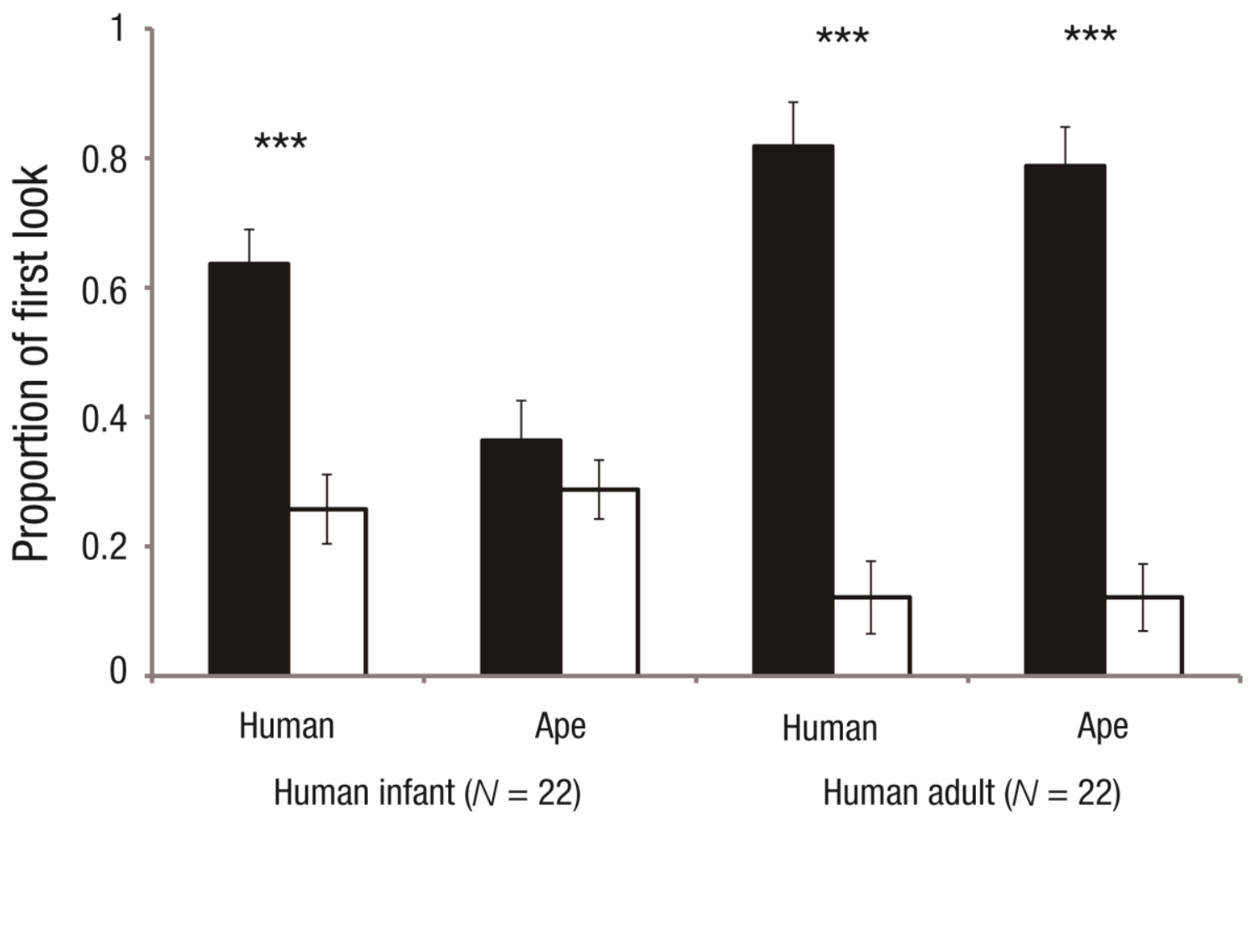


$\square$ Target

$\square$ Distractor

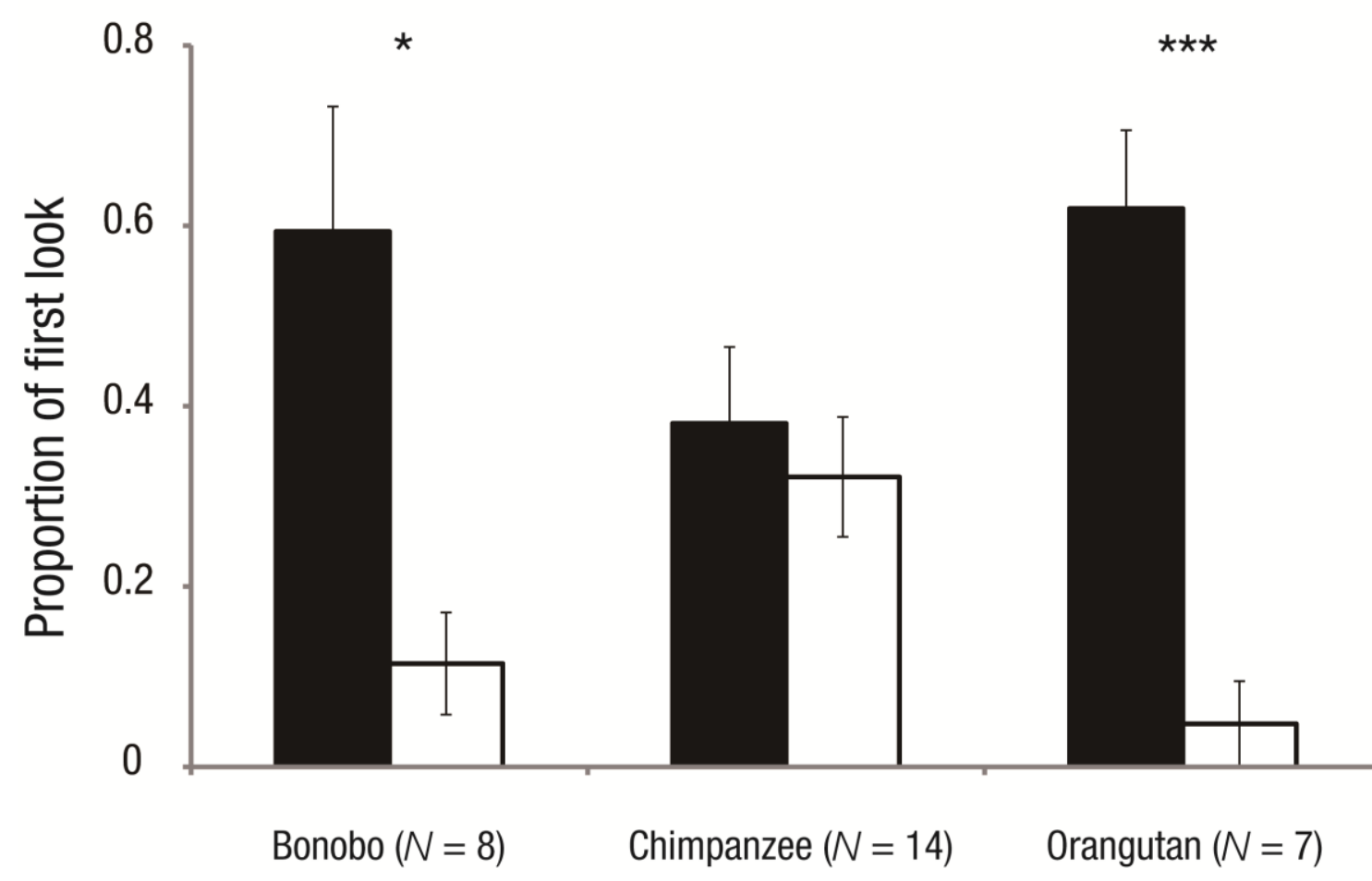

